\author{
Singhal Surinder $\mathrm{K}$ \\ Verma Hitesh \\ Dass Arjun \\ Punia Rajpal
}

Department of ENT \& Head Neck

Surgery

Government Medical College Hospital,

Chandigarh, India.

Correspondance to:

Dr. Surinder K Singhal

Dept. of ENT \& Head Neck Surgery, Government Medical College Hospital, Chandigarh, India

email: singhalsks@yahoo.com

\section{VALLECULAR CYSTS IN ADULT POPULATION: TEN YEAR EXPERIENCE}

Aims \& Objective:

To compare the results of LASER and cold knife surgery for the treatment of vallecular cysts in adults.

Material \& Methods:

It was an retrospective study of 24 consecutive patients who underwent LASER and cold knife surgery for vallecular cysts in university affiliated teaching hospital.

\section{Results:}

Three patients did not have adequate follow up \& hence were excluded from the study. Patients were assessed in follow up for any recurrences. Out of 21 patients, sixteen were male and five were females. Age ranged from $14-56$ years and duration of symptoms was $1-14$ months. All the patients revealed a cyst in the vallecula on indirect laryngoscopic examination and plain X-ray soft tissue neck lateral view. CT scan was done in two cases only. Tracheostomy was done in three patients for airway management (LASER-1,Cold Knife-2). Fifteen patients underwent LASER surgery where as six patients underwent cold knife surgery. Lingual surface of epiglottis was the most common site of origin (71\%). Follow up ranged from 6 months to 48 months. Two recurrences were noted in cold knife surgery group, whereas, no recurrence was noted in patients undergoing LASER surgery.

Conclusion:

LASER is a promising tool for treatment of vallecular cysts.

Key words: Vallecular cysts, LASER, Tracheostomy, Epiglottic cysts

\section{INTRODUCTION:}

Cystic lesions are common entities among the benign lesions in the laryngopharynx. These are usually retention cysts of the minor salivary glands and present at any age. Increase in the size of the cyst distorts the anatomy of laryngopharynx. Although often asymptomatic in adults, cysts can cause stridor, dyspnea, and feeding difficulties in infants and young children. Symptomatic cysts are typically treated by transoral marsupilization under microscopic, endoscopic, or direct visualization with cold instruments or LASER. Aims and objective of this study was to report our experience of treating cases of vallecular cysts in adults as a retrospective review and compare the results of LASER and cold knife surgery.

\section{MATERIAL AND METHODS:}

The retrospective study included cases of vallecular cysts who had presented to outpatient department of Otolaryngology, Head and Neck Surgery at Government Medical College and Hospital, Chandigarh from 2000-2010. A total of 24 patients of vallecular cysts were treated during this period. The patients were offered to choose for LASER or cold knife surgery after explaining the advantage \& disadvantage of both procedures. LASER was selected as modality of treatment as they felt it to be new and better modality.

All the patients underwent a detailed history and clinical examination including indirect laryngoscopy at the time of presentation. They were subsequently subjected to $X$ - ray soft tissue neck lateral view which revealed a soft tissue mass in the vallecula with varying degree of epiglottic shift and narrowing of airway (Fig.1). Computerized tomography was done in 2 cases as the indirect laryngoscopic findings in these patients were suspicious. All patients were operated under general anaesthesia. Consent for tracheostomy was taken from all the patients. In all the cases, awake intubation was done to prevent the laryngeal obstruction. Tracheostomy was done in three cases. In one case, it was done for difficult intubation and in other two cases for post-operative oedema. Fifteen patients were operated using LASER and six patients were done with cold knife method.

Under endotracheal general anaesthesia, Boyle Davis mouth gag was inserted to visualize the cyst in the vallecula. Throat packing was done in order to prevent spillage of the contents of the cyst in case it is ruptured. The cyst was held firmly with the help of tonsil holding forceps and dissected from the vallecula and lingual surface of the

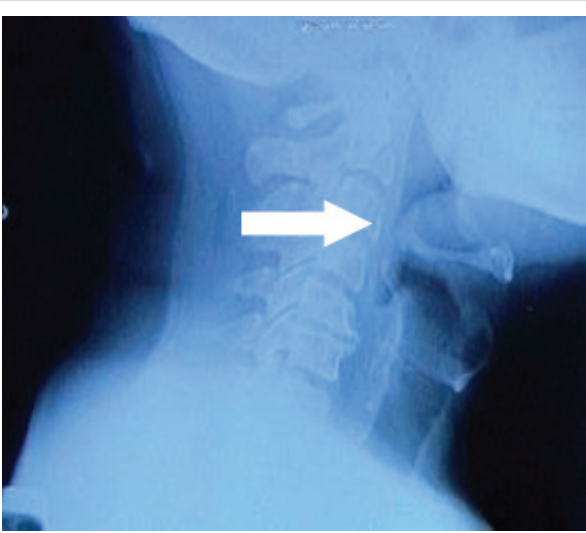

Fig.1. X-Ray soft tissue neck Lateral view showing cyst in the vallecula (White arrow)

epiglottis leaving the epiglottic cartilage bare. A Ryle's tube was inserted for feeding in the post-operative period. Two patients were tracheostomised as they developed respiratory distress after extubation. All the patients were closely observed in the postoperative period for any respiratory distress. They were given antibiotics steroids and anti$\mathrm{n} f \mathrm{I}$ a $\mathrm{m}$ m a tory analgesic drugs. Ryle's tube was removed after 48 hours. and patient was allowed liquids and semi solids orally. Tracheostomy which was done in two cases was closed after two weeks \& there was complete healing in four to six weeks.

In LASER surgery group patients were also taken up under general anaesthesia. The tube was wrapped with noninflammable material as we did not have the special tubes used for LASER surgery. The cyst was exposed in a similar way with help of Boyle Davis mouth. Wet sponges were kept in the perioral area with double protection for the eyes. All the instruments around the operative area were also protected with wet sponges to prevent LASER associated complications. In few patients exposure was not adequate, wide mouth direct laryngoscope was inserted to visualize the cyst. The laryngoscope was fixed with laryngeal suspension to keep both the hands free for instrumentation. LASER beam was focused with the help of microscope, $400 \mathrm{~mm}$ objective lens \& LASER power was set to $10 \mathrm{~W}$, continuous mode. In patients where Boyle Davis mouth gag was used LASER was delivered through hand applicator (Fig.2). 


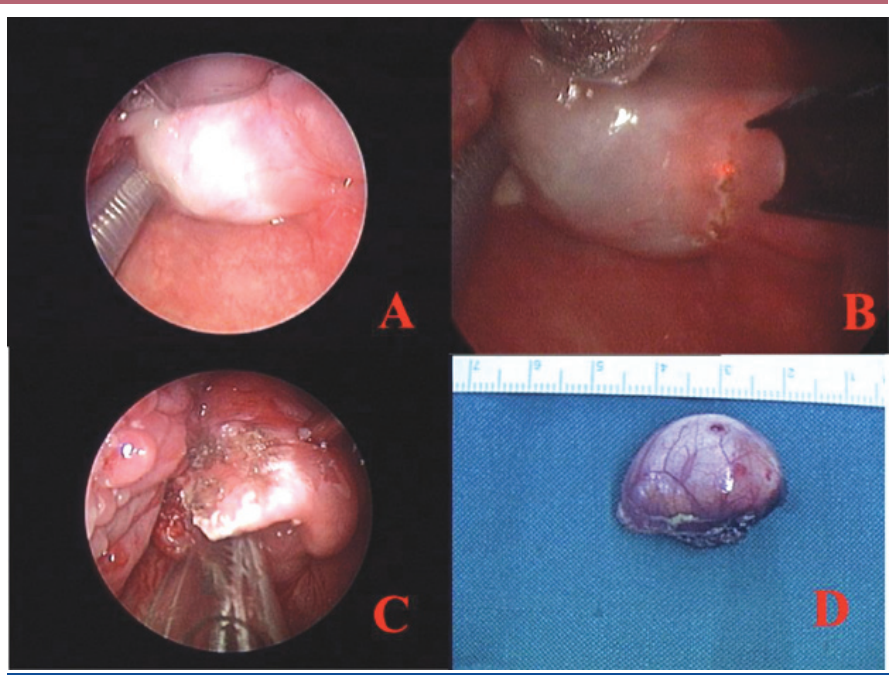

Fig.2: Photograph showing: A) Cyst in the vallecula, endotracheal tube also seen in picture, B) LASER hand applicator (White arrow) with focused beam (red spot) and end of Boyle Davis mouth gag (Blackarrow), C) Immediate post- operative view, (D) Excised cyst

With the hand applicator, LASER beam was used to dissect the cyst from the vallecula and lingual surface of the epiglottis as was done in cold knife surgery method. In case of bigger cysts where we could not locate the site of origin, it was punctured with LASER beam at one point and contents were sucked. After controlled decompression, one end of the cyst wall was held with a forceps and whole cyst was excised. Ryle's tube was inserted for feeding in post-operative period and was removed after 48 hour.

\section{RESULTS:}

24 Patients were treated during a period of 10 years. Three patients were excluded from the study as they did not come for follow up. Out of 21 patients studied, sixteen were male and five females. The age ranged from 32 to 60 years average being 46 years. The history of feeling of foreign body or lump was present in all the patients. Other symptoms were change in voice $14(66.6 \%)$ and choking $2(9.52 \%)$ of the patients. Indirect laryngoscopy revealed a cyst in the vallecula with shining surface in all the patients. Three patients were asymptomatic and were diagnosed as incidental finding on plain Xray soft tissue of the neck done for other purposes. The duration of symptoms ranged from 1 to 14 months. No patient presented with respiratory distress requiring urgent tracheostomy.

The duration of follow-up ranged from 6 months to 96 months. Recurrence was seen in 2 cases $(33.3 \%)$, out of six cases treated by cold knife method. The post-operative follow up revealed a mucosa lined vallecula and some evidence of fibrosis at the lateral glossoepiglottic fold area. In cases where the cyst was arising from the lateral free edge of the epiglottis, part of the epiglottis was excised and resulting defect was observed in follow up examination. There was no recurrence in cases treated by $\mathrm{CO} 2$ Laser.

Out of 21 cases lingual surface of the epiglottis was the commonest site of origin in $13(61.9 \%)$ cases. This was followed by aryepiglottic fold $5(23.8 \%)$, base of tongue $2(9.5 \%)$ and $1(4.7 \%)$ from lateral wall of vallecula. Histopathological examination (Fig.3) showed mucus retention cyst in 17 cases (80.9\%), lymphoepithelial cyst two cases and myxolipoma and squamous cell carcinoma in one each

\section{DISCUSSION:}

Vallecular cyst also known as epiglottic cyst or mucus retention cyst is classified as ductal cyst as a result of obstruction of the ducts of sub mucosal glands. They constitute about $5 \%$ of benign lesions of the larynx. 1 Of the laryngeal cyst, vallecular cysts accounts for $10.5 \%$ to $20.1 \%$ of all laryngeal cysts. 2 The most common location of epiglottic cyst is lingual surface of the epiglottis. DeSanto et al reviewed 238 cases of laryngeal cysts over a period of 10 years and found that 134 $(52 \%)$ were arising from the epiglottis3. These cysts gradually increase in size and fill up the vallecula. Literature review reveals that there is bimodal age of presentation. The peak incidence in adults is seen in $5^{\text {th }}$ and 6th decade and majority of the patients are males.4,5 The symptoms vary depending upon the age of the patient, site of the cyst and size of the cysts. In neonatal period the cysts have been reported to cause noisy and labored breathing along with feeding difficulties. The other presentation in children is failure to thrive. $4,6,7,8$ The other association in many of these children is laryngomalacia which further compounds the problem. The cysts in adults are usually asymptomatic and may be found as an incidental finding on X-ray or at the time of intubation for some surgical procedure. 9 Nearly $2 / 3$ rd of the cysts are asymptomatic.6 The other symptoms in adults are change in voice which is muffled, foreign body sensation, dysphagia and choking episodes. 10 Seventeen patients had a feeling of foreign body or lump in the throat, six patients had change in voice and two patients had choking episodes during night. Su CY and HSU JL also reported the similar symptomatology with feeling of lump in 23 patients and change in voice in 5 patients. 10 As the symptomatology varies with the age of presentation so is the method of investigation. In neonates and children flexible endoscopy may be required which is confirmatory in almost all the cases where as in adults the cysts are visible on indirect laryngoscopy. The cyst appears as shiny white mass in the vallecula with prominent vasculature over it. In patients having a strong gag reflex, $4 \%$ Xylocaine spray usually helps. Additionally rigid laryngoscope \& camera can be used in OPD to visualize the cyst and record the pictures for documentation and also for postoperative comparison. Unusual symptoms like dysphagia and haemoptysis has also been reported in the litrature. 11 The radiological investigations include plain X-ray soft tissue neck which demonstrates a soft tissue mass in the vallecula. (Fig.1) It is also helpful in knowing the degree of obstruction of the airway. CT scan can fairly demonstrate the mass in the vallecula and helps to differentiate it from the solid tumors. We did CT scan in two patients only where we were suspecting some other pathology. MRI has the best diagnostic effectiveness in demonstrating the smaller cysts but may be difficult in children \& neonates.12 The differential diagnoses include internal thyroglossal duct cysts, dermoid cysts, lingual thyroid, teratomas, lymphangiomas and haemangiomas. The management of vallecular cysts remains a challenge both in children and adults. Fiberoptic intubation without any relaxant is a good proposition but sometimes due to the large size of cyst and displacement of larynx it can be difficult. Direct laryngoscopy and intubation by an ENT surgeon can be rewarding in some cases. If this doesn't work, decompress the cyst after topical anaesthesia and intubate the patient. In neonates and children it has to be done carefully and quickly to avoid desaturation. However, tracheostomy always remains the choice when everything fails. We in our series, could intubate all the patients except one where we did tracheostomy. Another important thing is putting a pack in the glottis so as to avoid the aspiration of cystic fluid in case the cyst ruptures. The conventional modalities of management of cysts include marsupilization, de-roofing or excision. The complete surgical removal

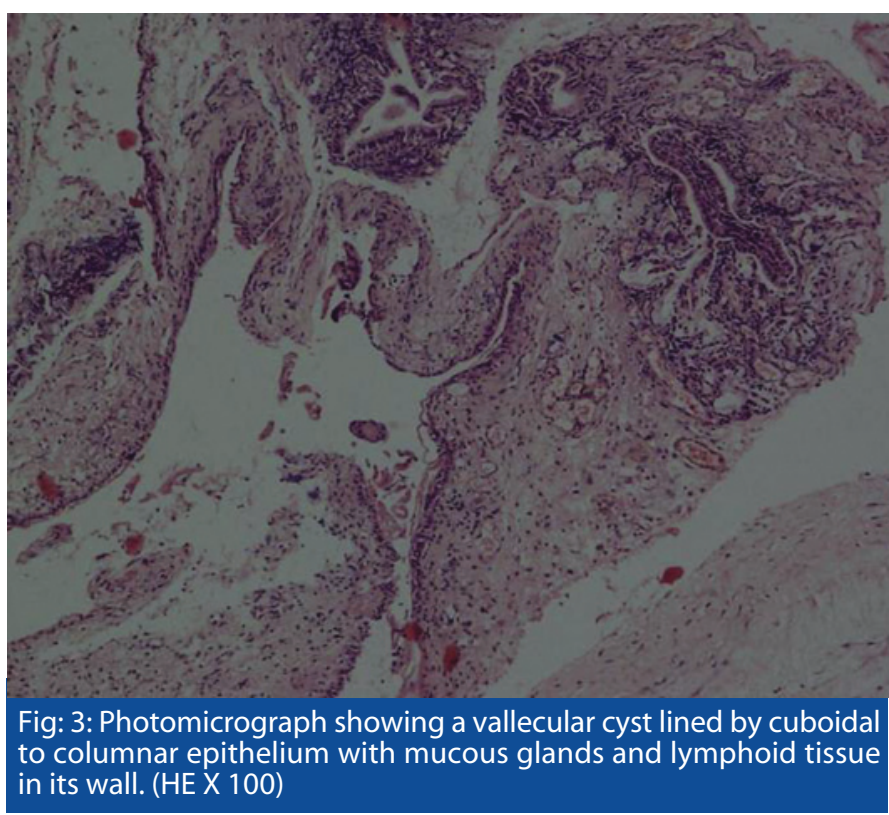


with laryngeal cold instrument is time consuming and difficult. We have observed in our patient that with cold instruments the tissue oedema is more because of the tissue handling and two of our patients required tracheostomy after extubation. The complete removal of cyst is proposed by most authors because simple aspiration of the content of the cyst is likely to results in recurrence12,13,14. Marsupilization and excision of cysts by LASER is new treatment modality. This technique allows excellent depth perception and good magnification, and permits more accurate assessment of the level of resection. It has the advantages of superior surgical precision, control and lower incidence of postoperative edema and pain 15 . However we, in our series, have removed the cysts in toto including the lining over the lingual surface of the epiglottis so as to reduce the chances of recurrence. In one of the case we resected part of the epiglottis as it was not looking like a typical cyst but was firm, attached to the epiglottis \& filling the vallecula. The biopsy in this case was myxolipoma. Histopatholgical reports in our series revealed majority of the lesions to be mucus retention cyst in 17 cases apart from two lymphoepithelial cyst, one each myxolipoma, and squamous cell carcinoma. This patient was subjected to radiotherapy and is without any recurrence till date. There was no recurrence in our series in patients treated with LASER where as two cases recurred in cold knife group. Simple aspiration of the cyst is associated with high recurrence.16 Suzuki et al reported one recurrence in 39 cases after marsupilization.13 On searching the Pubmed, we could not come across any study comparing the recurrence rates with Cold instruments and LASER. Su et al reported 28 patients where they did transoral LASER marsupilization of the vallecular cyst and did not find even a single recurrence. 11 However the literature is silent about the recurrence following the conventional surgery.

\section{CONCLUSIONS:}

LASER excision is the promising tool for the treatment of vallecular cyst.

\section{REFERENCES:}

1. Newman BH, Taxy JB, Laker HI. Laryngeal cysts in adults: a clinicopathologic study of 20 cases. Am J Clin Pathol. 1984 Jun;81(6):715-20.

2. Romak JJ, Olsen SM, Koch CA, Ekbom DC. Bilateral vallecular cysts as a cause of Dysphagia: case report and literature review. Int J Otolaryngol. 2010;2010: 697583 Epub 2010 Dec 12.
3. DeSanto LW, Devine KD, Werland LH (1970). Cyst of Larynx. Classification. Laryngoscope,80:397-400.

4. Gutiérrez JP, Berkowitz RG, Robertson CF. Vallecular cysts in newborns and young infants. Pediatric Pulmonology. 1999;27(4):282-285.

5. Arens $\mathrm{C}$, Glanz H, Kleinsasser O. Clinical and morphological aspects of laryngeal cysts. European Archives of Oto-Rhino-Laryngology. 1997;254(9-10):430-436).

6. Berger G, Averbuch E, Zilka K, Berger R, Ophir D. Adult vallecular cyst: thirteen-year experience. Otolaryngology. 2008;138 (3):321-327.

7. Tuncer U, Aydogan LB, Soylu L. Vallecular cyst: a cause of failure to thrive in an infant. Int J Pediatr Otorhinolaryngol. 2002 Sep 2:65(2): 133-5.

8. KuAS. Vallecular cyst: report of four cases -one with co-existing laryngomalacia. J. Laryngol Otol. March 2000; 114(3): 224-6.)

9. Kamble VA, Lilly RB, Gross JB. Unanticipated difficult intubation as a result of an asymptomatic vallecular cyst. Anaesthesiology 1999; 3:872-3.

10. Su CY, Hsu JL. Transoral LASERmarsupialization of epiglottic cysts. Laryngoscope. 2007 Jul; 117 (7):1153-4.

11. Alsaleh SA, Al-Ammar AY.Haemoptysis: a rare presentation of vallecular cyst. Saudi Med J. 2008 Oct; 29(10):1497-500)

12. Suzuki J, Hashimoto S, Watanabe K, Takahashi KCongenital vallecular cyst in an infant: case report and review of 52 recent cases. J Laryngol Otol. 2011 Jun 14:1-5.

13. Dahn MC, Panning B, Lenarz T. Acute apnoea cuased by an epiglottic cyst. Int.J Pediatr otorhinolarngol 1998:42;271-276,

14. Fang TJ, Cheng KS, Li HY. A huge epiglottic cyst causing airway obstruction in an adult. Chan Gung Med J 2002:25; 275-278.

15. Van de, Water FW. Laryngeal cysts: their surgical management. Laryngoscope 1973;83:1185-1194.

16. Chi Ying Su et al Transoral LASERmarsupialization of Epiglottic Cysts. Laryngoscope117:1153-1154, 2007.

17. Mitchell D, Irwin B, Bailey C et al. Cysts of the Infant larynx . J Laryngol Otol 1987:101;833-7 